\title{
Triaxially deformed freely precessing neutron stars: continuous electromagnetic and gravitational radiation
}

\author{
Yong Gao ${ }^{\circledR}, 1,2$ Lijing Shao ${ }^{\circledR}, 2,3 \star$ Rui Xu ${ }^{\circledR}, 2$ Ling Sun ${ }^{\circledR},{ }^{4,5}$ Chang Liu ${ }^{\circledR 1,2}$ and Ren-Xin Xu ${ }^{\circledR 1,2}$ \\ ${ }^{1}$ Department of Astronomy, School of Physics, Peking University, Beijing 100871, China \\ ${ }^{2}$ Kavli Institute for Astronomy and Astrophysics, Peking University, Beijing 100871, China \\ ${ }^{3}$ National Astronomical Observatories, Chinese Academy of Sciences, Beijing 100012, China \\ ${ }^{4}$ LIGO Laboratory, California Institute of Technology, Pasadena, CA 91125, USA \\ ${ }^{5}$ Centre for Gravitational Astrophysics, College of Science, The Australian National University, Acton, ACT 2601, Australia
}

Accepted 2020 August 13. Received 2020 July 31; in original form 2020 July 6

\begin{abstract}
The shape of a neutron star (NS) is closely linked to its internal structure and the equation of state of supranuclear matters. A rapidly rotating, asymmetric NS in the Milky Way undergoes free precession, making it a potential source for multimessenger observation. The free precession could manifest in (i) the spectra of continuous gravitational waves (GWs) in the kilohertz (kHz) band for ground-based GW detectors, and (ii) the timing behaviour and pulse-profile characteristics if the NS is monitored as a pulsar with radio and/or X-ray telescopes. We extend previous work and investigate in great detail the free precession of a triaxially deformed NS with analytical and numerical approaches. In particular, its associated continuous GWs and pulse signals are derived. Explicit examples are illustrated for the continuous GWs, as well as timing residuals in both time and frequency domains. These results are ready to be used for future multimessenger observation of triaxially deformed freely precessing NSs, in order to extract scientific implication as much as possible.
\end{abstract}

Key words: gravitational waves - methods: analytical - pulsars: general.

\section{INTRODUCTION}

Pulsars are magnetized rotating neutron stars (NSs). Using the socalled pulsar timing technique, the Hulse-Taylor pulsar provided the first validation for the existence of gravitational waves (GWs; Taylor, Fowler \& McCulloch 1979). In the new era after the direct observation of GWs with ground-based laser interferometric detectors (Aasi et al. 2015; Acernese et al. 2015; Abbott et al. 2016, 2017b, 2019a), pulsars continue to play an important role in the context of GW astrophysics. They can be perceived as GW sources radiating continuous GWs, as well as GW detectors in the form of pulsar timing arrays (Janssen et al. 2015; Perera et al. 2019). In this work, we are interested in freely precessing, asymmetric NSs, which can produce both modulated pulse signals and continuous GW radiations with characteristic features (Zimmermann \& Szedenits 1979; Zimmermann 1980; Bisnovatyi-Kogan, Mersov \& Sheffer 1990; Jones \& Andersson 2001, 2002), and thus become potential multimessenger sources of great scientific interest.

The most compelling evidence for NS free precession comes from PSR B 1828-11. Timing observation over 13 yr for this isolated pulsar showed strong Fourier power at periods of about 250, 500, and $1000 \mathrm{~d}$ (Stairs, Lyne \& Shemar 2000), which could be an indication for free precession. Link \& Epstein (2001) suggested the period at $500 \mathrm{~d}$ as the precession period of a biaxial NS with a precessing angle of $\sim 3^{\circ}$ and a dipole moment nearly orthogonal to the symmetric axis. They also interpreted the period at $250 \mathrm{~d}$ as a result of the electromagnetic dipole

^E-mail: 1shao@pku.edu.cn torque. Timing data of another pulsar, PSR B1642-03, provided additional support for the idea of NS free precession (Cordes 1993; Shabanova, Lyne \& Urama 2001). Recently, the Canadian Hydroden Intensity Mapping Experiment/Fast Radio Bursts (CHIME/FRB) Collaboration reported the detection of a $16.35 \pm 0.15$ d periodicity in the radio burst from FRB 180916.J0158+65 (Amiri et al. 2020). This periodicity may arise from the free precession of a magnetar (Levin, Beloborodov \& Bransgrove 2020; Zanazzi \& Lai 2020). Another possible evidence of precession comes from oscillations in gammaray burst afterglows. The afterglow may be powered by a long-lived remnant (Dai \& Lu 1998; Zhang et al. 2006), which might be a millisecond magnetar (Suvorov \& Kokkotas 2020). In the early stages of its life, the magnetar is likely to precess and result in modulation of X-ray luminosity. Suvorov \& Kokkotas (2020) found that the data of two bursts are highly consistent with precessing oblique rotators. While more evidence is needed to solidify the phenomenon of NS free precession, it is nowadays certainly interesting to study it in the context of GW astrophysics.

The conventional model for NS structure consists of a liquid core and a thin solid crust. Jones \& Andersson (2001) conjectured that the core of a NS does not participate in the free precession. Assuming the crust-only precession, they constructed a simple model with a thin radio beam fixed on the body of a biaxially deformed NS, and they assumed that the beam is aligned with the dipole moment. The model was applied to some potential candidates that may be undergoing free precession (Jones \& Andersson 2001). Although our understanding related to NSs has advanced remarkably (Baym et al. 2018), NS structure is still unclear and alternative models have already been proposed. NSs could actually be strange stars if Witten's conjecture 
is correct (Witten 1984), and they could globally be in a solid state if quarks are condensed in position space (Xu 2003) or momentum space (Mannarelli, Rajagopal \& Sharma 2007), resulting in highly elastic quadrupole deformations (Owen 2005) and thus large free precession amplitudes. Therefore, a multimessenger study of freely precessing NSs would help in understating the equation of state of cold matter at supranuclear density and distinguish between different models.

Previous studies dominantly focused on biaxial NSs. In the most generic case, the deformation of a NS does not need to be biaxial. A triaxially deformed NS can demonstrate new features in its free precession. We extend the simple model in Jones \& Andersson (2001) and study the timing residual of a freely precessing triaxial NS in this work. The internal dissipation from the frictional-type coupling between the crust and the core may damp the wobble angle in a relatively short time-scale (Jones \& Andersson 2002). As an illustrative work, we do not consider the damping here, but use different wobble angles in our calculation, from large ones to small ones, to display the modulations of spin period and spin period derivative in both time and frequency domains. The precession modulates the pulse width as well, which provides a good way to probe the beam shape of the pulsar radiation (Link \& Epstein 2001; Desvignes et al. 2019). We use a simple cone model (Gil, Gronkowski \& Rudnicki 1984; Lorimer \& Kramer 2005) to study the pulse-width modulation of triaxially deformed freely precessing NSs, and investigate the change of pulse width with different choices of wobble angles.

From the GW perspectives, precessing NSs have been recognized as potential sources of continuous GWs for decades (Zimmermann \& Szedenits 1979; Zimmermann 1980; Alpar \& Pines 1985). In the new era of GW astronomy, the detection of GWs from precessing NSs with ground-based detectors is imminent. Using the Advanced Laser Interferometer Gravitational-Wave Observatory (LIGO) data from its first and second observing runs, the search of continuous GWs at once and twice rotation frequencies from 222 pulsars has been performed (Abbott et al. 2019b). Stringent upper limits are set on the GW amplitude, the fiducial ellipticity, and the mass quadrupole moment via the search at the twice of the rotation frequency. These results can be used for testing various alternatives to the general relativity (GR; e.g. Xu, Zhao \& Shao 2020).

Zimmermann (1980) treated precessing triaxial NSs as rigid bodies and derived the quadrupole waveform for them. In addition, he simplified the waveform assuming a small wobble angle, and showed that the spectral lines of the continuous GWs are located at angular frequencies of $\Omega_{\mathrm{r}}+\Omega_{\mathrm{p}}$ and $2 \Omega_{\mathrm{r}}$, where $\Omega_{\mathrm{r}}$ is the rotation angular frequency, and $\Omega_{\mathrm{p}}$ is the free precession angular frequency of the NS. The first-order spectral lines yield little information about other physical properties of NSs beyond the rotation and precession frequencies. Based on Zimmermann (1980), Van Den Broeck (2005) obtained a third angular frequency at $2\left(\Omega_{\mathrm{r}}+\Omega_{\mathrm{p}}\right)$ by expanding the waveform to the second order of the wobble angle. The feasibility to detect continuous GWs from precessing triaxial NSs was reexamined, and it is found that the deviation from axisymmetry, the oblateness, and the wobble angle can be determined if the secondorder line is observed (Van Den Broeck 2005).

Following Zimmermann (1980) and Van Den Broeck (2005), in this work we use a Newtonian treatment for the precession, augmented with the GW radiation formalism in GR (Misner, Thorne \& Wheeler 1973). In this problem, the Newtonian treatment is indeed also valid for strong-field objects like NSs, if the GR expressions for the integrals of various moments are used (Thorne 1980). Similarly to Van Den Broeck (2005), we expand the GW

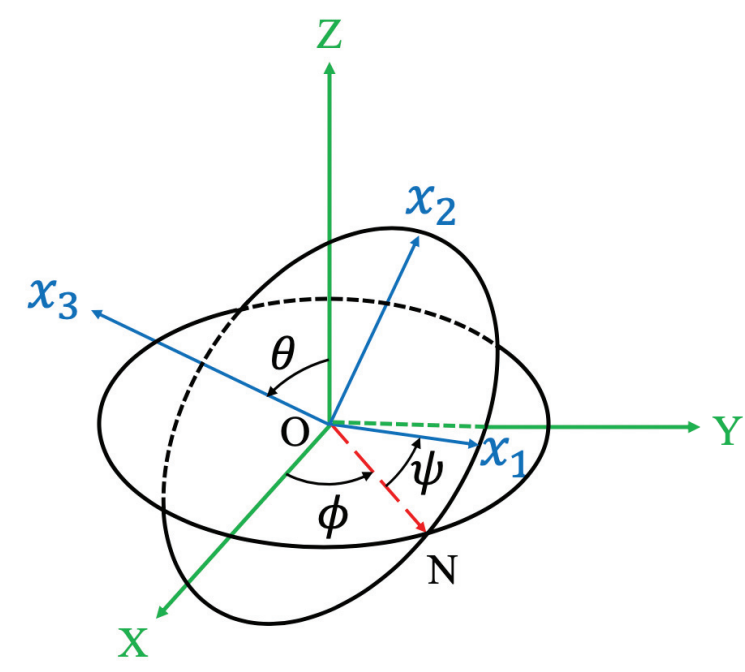

Figure 1. The inertial and body coordinate systems for the rigid body. Uppercase letters, X, Y, and Z, denote the inertial frame coordinates, while lowercase letters, $x_{1}, x_{2}$, and $x_{3}$, denote the coordinates in the body frame. Three Euler angles, $\theta, \phi$, and $\psi$, are defined as shown.

waveform to the second order of the precessing angle. However, unlike Van Den Broeck (2005), where a hierarchy for small parameters is assumed, more generically we treat the deviation from axisymmetry as a small parameter independent of the wobble angle in the expansion. Consequently, we obtain three more frequencies in the continuous GW spectra that are useful for a more complete extraction of physical information.

The structure of this paper is as follows. In Section 2, we provide both analytical and numerical solutions for freely precessing triaxial rigid bodies. Estimations of the oblateness, the non-axisymmetry, and the wobble angle for elastically deformed NSs are given based on existing literature. In Section 3, we show the timing residuals and pulse-width modulations of precessing triaxial NSs. These features could be identified if the NS is observed as a radio and/or X-ray pulsar. In Section 4, after briefly reviewing the quadrupole formula in Zimmermann (1980), we expand the waveform to the second order assuming a small wobble angle, a small non-axisymmetry, and a small oblateness. Because of the relaxation in the assumption about the small quantities, three new spectral lines are obtained with respect to previous studies. In Section 5, we discuss the extraction of physical information of NSs from radio signals and continuous GWs. We briefly summarize our work in Section 6.

\section{FREE PRECESSION OF TRIAXIAL RIGID BODIES}

In general, the rotation axis of a rigid body does not coincide with its principal axes. As a consequence, a freely rotating rigid body precesses around the direction of the total angular momentum (Landau \& Lifshitz 1960). The motion of the body can be described by three Euler angles, $\theta, \phi$, and $\psi$, and their time derivatives. In Fig. 1, we denote the coordinates of the inertial reference frame by uppercase letters, $\mathrm{X}, \mathrm{Y}$, and $\mathrm{Z}$, with unit basis vectors $\widehat{e}_{\mathrm{X}}, \widehat{e}_{\mathrm{Y}}$, and $\widehat{e}_{\mathrm{Z}}$. The vector $\widehat{e}_{\mathrm{Z}}$ is chosen to be in the direction of the angular momentum of the rigid body, $\boldsymbol{J}$. We use lowercase letters, $x_{1}, x_{2}$, and $x_{3}$, to denote the coordinates in the body frame, which is attached on the rigid body. Their unit basis vectors are $\widehat{e}_{1}, \widehat{e}_{2}$, and $\widehat{e}_{3}$, chosen to be parallel to the three individual eigenvectors of the moment of 
inertia tensor. We use $I_{1}, I_{2}$, and $I_{3}$ as the diagonal components of the moment of inertia tensor in the body frame.

For freely precessing rigid bodies, the dynamical equations of motion in the body frame are (Landau \& Lifshitz 1960)

$I_{1} \dot{\omega}_{1}-\left(I_{2}-I_{3}\right) \omega_{2} \omega_{3}=0$,

$I_{2} \dot{\omega}_{2}-\left(I_{3}-I_{1}\right) \omega_{3} \omega_{1}=0$,

$I_{3} \dot{\omega}_{3}-\left(I_{1}-I_{2}\right) \omega_{1} \omega_{2}=0$,

where $\omega_{1}, \omega_{2}$, and $\omega_{3}$ represent the angular velocities along $\widehat{e}_{1}$, $\widehat{e}_{2}$, and $\widehat{e}_{3}$. The dots denote the derivatives with respect to time $t$. Considering the kinematics of the rigid body, the evolution of the three Euler angles can be described by (Landau \& Lifshitz 1960)

$\omega_{1}=\dot{\phi} \sin \theta \sin \psi+\dot{\theta} \cos \psi$,

$\omega_{2}=\dot{\phi} \sin \theta \cos \psi-\dot{\theta} \sin \psi$,

$\omega_{3}=\dot{\phi} \cos \theta+\dot{\psi}$,

where the Euler angles are defined in Fig. 1.

As the rigid body is torque free, both the kinetic energy,

$E=\frac{1}{2}\left(I_{1} \omega_{1}^{2}+I_{2} \omega_{2}^{2}+I_{3} \omega_{3}^{2}\right)$,

and the angular momentum,

$J=\left(I_{1}^{2} \omega_{1}^{2}+I_{2}^{2} \omega_{2}^{2}+I_{3}^{2} \omega_{3}^{2}\right)^{1 / 2}$,

are conserved. In the following, we assume that the principal moments of inertia satisfy $I_{1}<I_{2}<I_{3}$. We also assume $J^{2}>$ $2 E I_{2}$, which is equivalent to the condition that the tail of the angular momentum $\boldsymbol{J}$ moves around $\widehat{e}_{3}$ along a closed curve in the body frame (Landau \& Lifshitz 1960). Results for other choices can be obtained by properly relabeling the indices.

The motion of a rigid body described by equations (1)-(6) is an initial value problem. In principle, one can obtain the evolution of the orientation of the triaxial rigid body at any time once the initial values of the three Euler angles and the angular velocities are specified. In subsequent calculations, we choose the initial values such that at $t=$ 0 , one has $\phi=0, \psi=\pi / 2$, and $\theta$ is at its minimum value $\theta_{\min }$. The initial values of the angular velocities in the body frame are denoted as $\omega_{1}=a, \omega_{2}=0$, and $\omega_{3}=b$ at $t=0$. These assumptions can easily be extended to generic cases. Worth to stress that, throughout the calculation, we have assumed that the moments $I_{i}(i=1,2,3)$ are constant and the body undergoes free precession. We have ignored the damping of the precession due to some physical processes for NSs (e.g. the fluid dynamics of the NS interior). Now, we discuss the analytical solution and the numerical method to solve the equations of motion in equations (1)-(6).

\subsection{Analytical solution}

The exact analytical solution to equations (1)-(6) for a precessing triaxial rigid body has been obtained in terms of the elliptic functions (Landau \& Lifshitz 1960; Zimmermann 1980; Whittaker 1988; Shakura, Postnov \& Prokhorov 1998; Van Den Broeck 2005; Akgun, Link \& Wasserman 2006; Lasky \& Melatos 2013; Pina 2015). Here we briefly review the solution according to Landau \& Lifshitz (1960) for readers' convenience.

The angular velocities in the body frame are

$\omega_{1}(\tau)=a \operatorname{cn}(\tau, m)$,
$\omega_{2}(\tau)=a\left[\frac{I_{1}\left(I_{3}-I_{1}\right)}{I_{2}\left(I_{3}-I_{2}\right)}\right]^{1 / 2} \operatorname{sn}(\tau, m)$,

$\omega_{3}(\tau)=b \operatorname{dn}(\tau, m)$,

where $\tau$ is the dimensionless time variable,

$\tau=t \sqrt{\frac{\left(I_{3}-I_{2}\right)\left(J^{2}-2 E I_{1}\right)}{I_{1} I_{2} I_{3}}}$,

and sn, cn, and dn are the elliptic functions (see e.g. Olver et al. 2010). The parameter $m$ can be expressed as

$m=\frac{\left(I_{2}-I_{1}\right) I_{1} a^{2}}{\left(I_{3}-I_{2}\right) I_{3} b^{2}}$

The angular velocities in the body frame are periodic with a period,

$T=\frac{4 K(m)}{b}\left[\frac{I_{1} I_{2}}{\left(I_{3}-I_{1}\right)\left(I_{3}-I_{2}\right)}\right]^{1 / 2}$,

where $K(m)$ is the complete elliptic integral of the first kind (Olver et al. 2010). The period $T$ is the free precession period. If $I_{1}$ is nearly equal to $I_{2}$, the parameter $m$ is close to zero. In this case, the period $T$ is approximately $2 \pi I_{1} /\left[\omega_{3}\left(I_{3}-I_{1}\right)\right]$, which is the well-known free precession period for a biaxial body.

The Euler angles $\theta$ and $\psi$ are also periodic, and can be expressed as

$$
\begin{aligned}
& \cos \theta=\frac{I_{3} b}{J} \operatorname{dn}(\tau, m), \\
& \tan \psi=\left[\frac{I_{1}\left(I_{3}-I_{2}\right)}{I_{2}\left(I_{3}-I_{1}\right)}\right]^{1 / 2} \frac{\mathrm{cn}(\tau, m)}{\operatorname{sn}(\tau, m)} .
\end{aligned}
$$

From the above two equations, one finds that the angle $\theta$ has a period of $T / 2$, while the angle $\psi$ has a period of $T$. In contrast, the angle $\phi$ is not periodic. It can be represented as a sum of two parts, $\phi=\phi_{1}$ $+\phi_{2}$. The 'periodic' part $\phi_{1}$ has a period of $T / 2$, and is defined via

$\exp \left[2 \mathrm{i} \phi_{1}(t)\right]=\frac{\vartheta_{4}\left(\frac{2 \pi t}{T}+\mathrm{i} \pi \alpha, q\right)}{\vartheta_{4}\left(\frac{2 \pi t}{T}-\mathrm{i} \pi \alpha, q\right)}$,

where $\vartheta_{4}$ is the fourth Jacobi theta function with nome $q=$ $\exp [-\pi K(1-m) / K(m)]$. In equation (17), $\alpha$ is determined via

$\operatorname{sn}[2 \mathrm{i} \alpha K(m)]=\frac{\mathrm{i} I_{3} b}{I_{1} a}$.

The 'linear-in-time' part $\phi_{2}$ is given by

$\phi_{2}=\frac{2 \pi t}{T_{1}}=\left(\frac{J}{I_{1}}+\frac{2 \pi \mathrm{i}}{T} \frac{\vartheta_{4}^{\prime}(\mathrm{i} \pi \alpha, q)}{\vartheta_{4}(\mathrm{i} \pi \alpha, q)}\right) t$

where $\vartheta_{4}^{\prime}(u, q)$ is the derivative of $\vartheta_{4}(u, q)$ with respect to $u{ }^{1}$ As $I_{1}$ approaches $I_{2}$, the period $T_{1}$ in equation (19) approaches $2 \pi I_{1} / J$. Generally, $T$ and $T_{1}$ are not commensurate with each other, so the motion of the body is not periodic in the inertia frame. For simplicity, we define (Zimmermann 1980; Van Den Broeck 2005)

$\Omega_{\mathrm{p}} \equiv \frac{2 \pi}{T}=\frac{\pi b}{2 K(m)}\left[\frac{\left(I_{3}-I_{2}\right)\left(I_{3}-I_{1}\right)}{I_{1} I_{2}}\right]^{1 / 2}$,

${ }^{1}$ Note that the solution of the Euler angle $\phi$ in Landau \& Lifshitz (1960) and Zimmermann (1980) has sign typos when the following theta function and its derivative (Whittaker 1988) are used as they claimed. The fourth Jacobi theta function is defined as $\vartheta_{4}(u, q)=1+$ $2 \sum_{n=1}^{\infty}(-1)^{n} q^{n^{2}} \cos (2 \pi n u)$, and the derivative of $\vartheta_{4}$ with respect to $u$ is $\vartheta_{4}^{\prime}(u, q)=4 \pi \sum_{n=1}^{\infty} n(-1)^{n+1} q^{n^{2}} \sin (2 \pi n u)$. 
$\Omega_{\mathrm{r}} \equiv \frac{2 \pi}{T_{1}}-\frac{2 \pi}{T}=\frac{J}{I_{1}}+\frac{2 \pi \mathrm{i}}{T} \frac{\vartheta_{4}^{\prime}(\mathrm{i} \pi \alpha, q)}{\vartheta_{4}(\mathrm{i} \pi \alpha, q)}-\Omega_{\mathrm{p}}$

for later use.

\subsection{Numerical approach using quaternions}

Although the analytical solution given in the above subsection is exact, the use of it is not intuitive. Here we discuss a numerical method to integrate the equations of motion. There are two reasons for the need of a numerical method. First, numerical methods can avoid the use of the elliptic functions. Second, they can be easily applied to general equations of motion where precessions are much involved with torques in consideration. In generic cases with torques, analytical solutions usually do not exist.

In our numerical calculation, we employ the numerical method solving three-dimensional rotations through the use of quaternions, which is a mathematically equivalent formalism to the aforementioned one using the Euler angles in describing rotations in the threedimensional space (Arribas, Elipe \& Palacios 2006). The rotation matrix can be either written in terms of trigonometric functions of the Euler angles, or expressed by a specific quaternion whose time evolution is determined by the angular velocity of the rigid body. In numerical integrations, the latter is preferred because it produces stable results more efficiently (Arribas et al. 2006).

A quaternion, $q=q_{0}+q_{1} \boldsymbol{i}+q_{2} \boldsymbol{j}+q_{3} \boldsymbol{k}$, in the quaternion basis, $\{1, \boldsymbol{i}, \boldsymbol{j}, \boldsymbol{k}\}$, is usually denoted as

$q=\left(q_{0}, \boldsymbol{q}\right)$,

with $\boldsymbol{q}=\left(q_{1}, q_{2}, q_{3}\right)$ being a three-vector when used in calculating three-dimensional rotations. The rotation transformation from $r$ to $\boldsymbol{r}^{\prime}$ is performed via (Coutsias \& Romero 2004)

$$
\begin{aligned}
\left(0, \boldsymbol{r}^{\prime}\right) & =q(0, \boldsymbol{r}) \tilde{q} \\
& =\left(0,\left(q_{0}^{2}-\boldsymbol{q} \cdot \boldsymbol{q}\right) \boldsymbol{r}+2 \boldsymbol{q}(\boldsymbol{q} \cdot \boldsymbol{r})+2 q_{0} \boldsymbol{q} \times \boldsymbol{r}\right) \\
& =(0, \mathcal{R} \boldsymbol{r})
\end{aligned}
$$

where $\tilde{q}=\left(q_{0},-\boldsymbol{q}\right)$ is the conjugate quaternion of $q$. Note that from the first to the second line we have used the multiplication rule of quaternions, and from the second to the third line the usual dot product and cross product of three vectors are applied. Explicitly, the rotation matrix $\mathcal{R}$ in equation (23) is

$\mathcal{R}=\left(\begin{array}{ccc}q_{0}^{2}+q_{1}^{2}-q_{2}^{2}-q_{3}^{2} & 2 q_{1} q_{2}-2 q_{0} q_{3} & 2 q_{1} q_{3}+2 q_{0} q_{2} \\ 2 q_{1} q_{2}+2 q_{0} q_{3} & q_{0}^{2}-q_{1}^{2}+q_{2}^{2}-q_{3}^{2} & 2 q_{2} q_{3}-2 q_{0} q_{1} \\ 2 q_{1} q_{3}-2 q_{0} q_{2} & 2 q_{2} q_{3}+2 q_{0} q_{1} & q_{0}^{2}-q_{1}^{2}-q_{2}^{2}+q_{3}^{2}\end{array}\right)$,

which equals the normal Euler rotation matrix. Comparing the elements of them, we can relate the quaternion $q$ with the Euler angles through

$$
\begin{aligned}
& q_{0}=\cos \frac{\theta}{2} \cos \left(\frac{1}{2}(\phi+\psi)\right), \\
& q_{1}=\sin \frac{\theta}{2} \cos \left(\frac{1}{2}(\phi-\psi)\right), \\
& q_{2}=\sin \frac{\theta}{2} \sin \left(\frac{1}{2}(\phi-\psi)\right), \\
& q_{3}=\cos \frac{\theta}{2} \sin \left(\frac{1}{2}(\phi+\psi)\right) .
\end{aligned}
$$

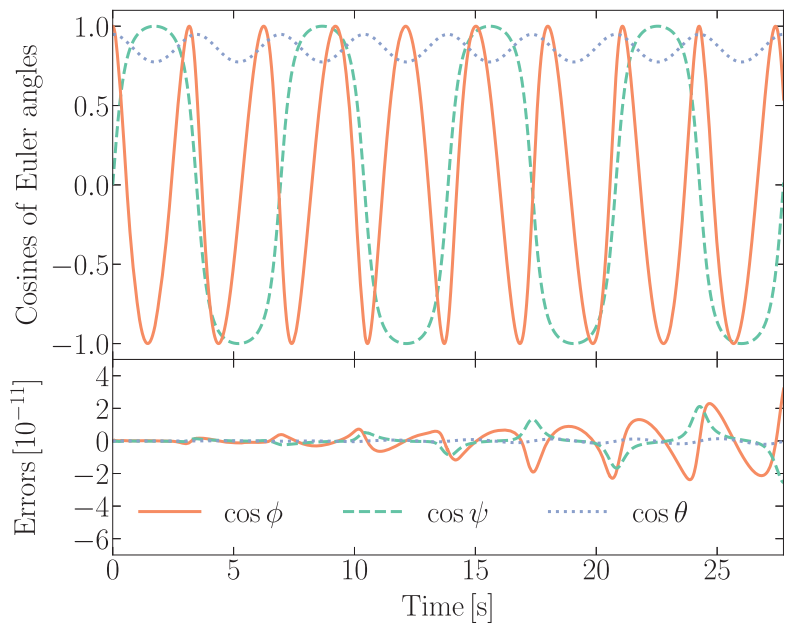

Figure 2. Upper panel: the numerically solved time evolution for cosines of $\theta, \psi$, and $\phi$. Lower panel: absolute error of the numerical results with respect to the analytical results for cosines displayed in the upper panel. In this plot, for illustrative purposes we have taken generic values for the rigid body: $I_{1} / I_{3}=1 / 3, I_{2} / I_{3}=2 / 3$, and $a=b=1 \mathrm{rad} \mathrm{s}^{-1}$. With these values, we have a free-precession period $T=6.9 \mathrm{~s}$. The figure contains four free precession periods.

In addition, the differential equation that governs the time evolution of the quaternion $q$ can be expressed as (Coutsias \& Romero 2004; Betsch \& Siebert 2009)

$\frac{\mathrm{d} q}{\mathrm{~d} t}=\frac{1}{2} q(0, \omega)=\frac{1}{2}\left(\begin{array}{cccc}0 & -\omega_{1} & -\omega_{2} & -\omega_{3} \\ \omega_{1} & 0 & \omega_{3} & -\omega_{2} \\ \omega_{2} & -\omega_{3} & 0 & \omega_{1} \\ \omega_{3} & \omega_{2} & -\omega_{1} & 0\end{array}\right)\left(\begin{array}{l}q_{0} \\ q_{1} \\ q_{2} \\ q_{3}\end{array}\right)$.

Once the initial orientation of the rigid body is given, one can translate it into the initial value of the quaternion $q$ using equations (25)-(28). Together with the initial values of $\omega_{1}, \omega_{2}$, and $\omega_{3}$, equations (1)-(3) and equation (29) can be integrated to obtain the angular velocities in the body frame and the time evolution of the quaternion $q(t)$, hence the elements of the rotation matrix $\mathcal{R}$ at any given time. The Euler angles can be recovered by the elements of the matrix $\mathcal{R}$ via

$$
\begin{aligned}
& \phi=\tan ^{-1}\left(-\frac{\mathcal{R}_{13}}{\mathcal{R}_{23}}\right), \\
& \theta=\tan ^{-1}\left(\frac{\sqrt{1-\mathcal{R}_{33}^{2}}}{\mathcal{R}_{33}}\right), \\
& \psi=\tan ^{-1}\left(\frac{\mathcal{R}_{31}}{\mathcal{R}_{32}}\right),
\end{aligned}
$$

where $\mathcal{R}_{i j}(i, j=1,2,3)$ is the component of the matrix $\mathcal{R}$. In Fig. 2, we present an explicit example, where the numerical result and the absolute error relative to the analytical solution are shown. Without dedicated efforts in obtaining Fig. 2, the numerical accuracy is already well below a few parts in trillion in this case. Note that this example is for a very generic case of free precession for a triaxially deformed rigid body, while the realistic situation for NSs is much milder as we are to discuss below, thus we expect significantly better numerical accuracy than what is shown in Fig. 2. 


\subsection{Physical parameters for triaxial NSs}

Before closing this section, let us discuss some typical values for relevant physical parameters of triaxial NSs. To describe the precession of triaxial NSs, one can define three small parameters out of $I_{1}, I_{2}, I_{3}, a$, and $b$, appearing in the previous equations. They are usually taken as the oblateness,

$\epsilon \equiv \frac{I_{3}-I_{1}}{I_{3}}$

the non-axisymmetry,

$\delta \equiv \frac{I_{2}-I_{1}}{I_{3}-I_{2}}$

and the tangent of the initial wobble angle,

$\gamma \equiv \tan \theta_{\min }=\frac{I_{1} a}{I_{3} b}$.

We first discuss the oblateness due to elastic deformations of NSs. For a conventional NS with a liquid core and a solid crust, the oblateness is (Baym \& Pines 1971; Jones \& Andersson 2001)

$\epsilon_{\text {elast }}=\eta \epsilon_{0}$,

where $\eta$ is the so-called rigidity parameter (Jones \& Andersson 2001), and $\epsilon_{0}$ is the zero-strain oblateness. The rigidity parameter $\eta$ is unity for a perfectly rigid star and zero for a liquid star. By assuming the shear modulus in the crust to be constant, the rigidity parameter for a NS with a liquid core can be approximated as (Baym \& Pines 1971)

$\eta \simeq \frac{57 \mu V_{\mathrm{c}}}{10\left|E_{\mathrm{g}}\right|} \simeq 2.3 \times 10^{-5}\left(\frac{\mu}{10^{30} \mathrm{erg} \mathrm{cm}^{-3}}\right) R_{6}^{4} M_{1.4}^{-2}$,

where $\mu$ is the shear modulus of the crust, $V_{\mathrm{c}}$ is the volume of the crust, and $E_{\mathrm{g}}=-3 G M^{2} / 5 R$ is the gravitational binding energy of the NS. The notations $M_{1.4}$ and $R_{6}$ represent the dimensionless NS mass $M_{1.4} \equiv M /\left(1.4 \mathrm{M}_{\odot}\right)$ and the dimensionless NS radius $R_{6} \equiv$ $R /\left(10^{6} \mathrm{~cm}\right)$. Cutler, Ushomirsky \& Link (2003) adopted a relativistic NS structure and solved for the strain field in the crust that evolves as the NS spins down. They found that $\eta$ is smaller than the estimation in Baym \& Pines (1971) by a factor of $\sim 40$. The estimation of the zero-strain oblateness $\epsilon_{0}$ is (Cutler et al. 2003; Van Den Broeck 2005)

$\epsilon_{0} \simeq \frac{\Omega_{\mathrm{r}}^{2} R^{3}}{G M}=2.1 \times 10^{-3}\left(\frac{f_{\mathrm{r}}}{100 \mathrm{~Hz}}\right)^{2} R_{6}^{3} M_{1.4}^{-1}$,

where $f_{\mathrm{r}}=\Omega_{\mathrm{r}} / 2 \pi$ is the spin frequency of the NS, and $\Omega_{\mathrm{r}}$ is defined in equation (21). Combining equations (36)-(38), we obtain the oblateness due to the elastic deformation,

$\epsilon_{\text {elast }} \simeq 4.9 \times 10^{-8}\left(\frac{f_{\mathrm{r}}}{100 \mathrm{~Hz}}\right)^{2}\left(\frac{\mu}{10^{30} \mathrm{erg} \mathrm{cm}^{-3}}\right) R_{6}^{7} M_{1.4}^{-3}$.

The oblateness for a NS due to elastic deformation is also limited by the breaking strain $\sigma_{\text {break }}$. According to Owen (2005), the largest oblateness is

$\epsilon_{\max }=3.4 \times 10^{-7}\left(\frac{\sigma_{\text {break }}}{10^{-2}}\right) \frac{M_{1.4}^{-2.2} R_{6}^{4.26}}{1+0.7 M_{1.4} R_{6}^{-1}}$.

The value of $\sigma_{\text {break }}$ is uncertain. Early estimations are in the range from $10^{-4}$ to $10^{-2}$ (Ruderman et al. 1992). Horowitz \& Kadau (2009) found that $\sigma_{\text {break }}$ is around 0.1 by simulating the crust as Coulomb solids. Recent semi-analytical lattice studies of Baiko \& Chugunov (2018) showed that $\sigma_{\text {break }}$ is more like to be 0.04 . Note that with this value of $\sigma_{\text {break }}$, the largest oblateness is about $\epsilon_{\max }=8 \times 10^{-7}$.
Now we turn to the non-axisymmetry. NSs are biaxial when $\delta$ is zero or infinity, and triaxial when $\delta$ has a finite value. Because of the complex evolution and relaxation of the crust after the star's birth and during the accretion in the late lifetime (Link, Franco \& Epstein 1998; Shakura et al. 1998; Link 2003; Akgun et al. 2006), deformed NSs are possible to be triaxial, characterized by finite values of $\delta$. The magnetic stresses might contribute to the triaxiality as well (Wasserman 2003). The non-axisymmetry depends on the evolution and relaxation of the crust and the magnetic stresses, which are complex, especially during dynamical or explosive processes. Because of our lack of knowledge about $\delta$, a measurement of it would be particularly exciting.

As for the wobble angle $\theta$, there is no physical limitation for slowly rotating NSs. However, for a fast rotating one, as the rotational bulge of the NS turns larger, more matter needs to be displaced during the precession, leading to a larger crust strain (Jones \& Andersson 2001; Van Den Broeck 2005). In order to keep the strain below the limit of $\sigma_{\text {break }}$, the star can only possess a small wobble angle. Jones \& Andersson (2001) estimated the maximum allowed wobble angle,

$\theta_{\max } \approx 0.45\left(\frac{100 \mathrm{~Hz}}{f_{\mathrm{r}}}\right)^{2}\left(\frac{\sigma_{\mathrm{break}}}{10^{-3}}\right) M_{1.4} R_{6}^{-3}$.

The constraint on the wobble angle depends on the rotation frequency and the breaking strain $\sigma_{\text {break }}$. For a NS with $f_{\mathrm{r}}=100 \mathrm{~Hz}$ and $\sigma_{\text {break }}=$ $10^{-3}$, the wobble angle is smaller than $0.45 \mathrm{rad}$. If we take the breaking strain in the extreme case where $\sigma_{\text {break }}=0.1$, the wobble angle is basically unlimited even for a fast rotating NS at a spin frequency of $f_{\mathrm{r}}=500 \mathrm{~Hz}$.

For the theoretical analysis in subsequent sections, we apply series expansion for the trigonometric functions of the three Euler angles in Section 2.1, assuming a small oblateness, a small non-axisymmetry, and a small wobble angle. The benefit of the perturbative treatment is the great simplification it brings and the explicit harmonics appearing in the spectra. As for generic cases when one or more of these parameters are large, one can always restore back to the exact solution (or the numerical scheme) for a careful check.

Following Zimmermann (1980) and Van Den Broeck (2005), we find that practically it is more convenient to use

$\kappa \equiv \frac{1}{16} \frac{I_{3}}{I_{1}} \frac{I_{2}-I_{1}}{I_{3}-I_{2}}$

than $\delta$ in the expansion. Up to the leading order, $\kappa$ and $\delta$ are related by $\kappa \simeq \delta / 16$. A constant of $1 / 16$ is included for the convenience of later computation (Van Den Broeck 2005). The parameter $m$ in equation (13) is then simplified to $m=16 \kappa \gamma^{2}$. In the series expansion, different from Van Den Broeck (2005), we treat $\gamma$ and $\kappa$ independently and do not assume any hierarchy between them. The series expansions of trigonometric functions of the three Euler angles up to the second order of $\gamma$ and $\kappa$ are

$$
\begin{aligned}
& \cos \phi= \cos \left[\left(\Omega_{\mathrm{r}}+\Omega_{\mathrm{p}}\right) t\right], \\
& \sin \phi=\sin \left[\left(\Omega_{\mathrm{r}}+\Omega_{\mathrm{p}}\right) t\right], \\
& \cos \theta=1-\frac{\gamma^{2}}{2}, \\
& \sin \theta=\gamma+8 \gamma \kappa \sin ^{2}\left(\Omega_{\mathrm{p}} t\right), \\
& \cos \psi=\sin \left(\Omega_{\mathrm{p}} t\right)\left[1+\left(8 \kappa+32 \kappa^{2}\right) \cos ^{2}\left(\Omega_{\mathrm{p}} t\right)\right] \\
&+\sin \left(\Omega_{\mathrm{p}} t\right)\left[16 \kappa^{2} \cos ^{2}\left(\Omega_{\mathrm{p}} t\right)\left(3 \cos \left(2 \Omega_{\mathrm{p}} t\right)+1\right)\right],
\end{aligned}
$$




$$
\begin{aligned}
\sin \psi= & \cos \left(\Omega_{\mathrm{p}} t\right)\left[1-\left(8 \kappa+32 \kappa^{2}\right) \sin ^{2}\left(\Omega_{\mathrm{p}} t\right)\right] \\
& -\cos \left(\Omega_{\mathrm{p}} t\right)\left[96 \kappa^{2} \sin ^{2}\left(\Omega_{\mathrm{p}} t\right) \cos ^{2}\left(\Omega_{\mathrm{p}} t\right)\right] .
\end{aligned}
$$

In subsequent sections, we apply the analytical solution in equations (9)-(19) and above estimation for the oblateness, the non-axisymmetry, and the wobble angle to investigate the timing behaviour and the GW radiation of precessing triaxial NSs. Note that the internal, fluid-dynamical mechanisms that might reorient the NS over time are not included in the calculation. They should exist in general, but depend on the equation of state of NSs (see e.g. Xu 2003, for solid strange stars). In addition, the magnetic field evolution or pulsation will lead to time-dependent changes in the oblateness and the non-axisymmetry as well.

The braking torques (Goldreich 1970) and the gravitational radiation reaction (Cutler \& Jones 2001) are also omitted for simplicity, which will make the spin frequency decrease and change the wobble angle. For very young and/or highly magnetized NSs, this might be quite important. Magnetic pressures can significantly adjust the precession period and lead to a non-zero time derivative for the free precession angular frequency, $\dot{\Omega}_{\mathrm{p}}$, because the oblateness might evolve rapidly and the NS spins down much faster due to large spin-down torques (Levin et al. 2020; Suvorov \& Kokkotas 2020; Zanazzi \& Lai 2020). For some systems, the braking torques and the gravitational radiation reaction may become important over long time-scales. The details are beyond the scope of this paper and deserve further investigation.

\section{MODULATED TIMING AND PULSE SIGNALS}

If a triaxially deformed freely precessing NS is observed as a pulsar, the free precession will introduce characteristic modulations on the timing and pulse signals. These modulations might be revealed by radio and/or X-ray observations. In Section 3.1, we discuss the phase modulations of precessing triaxial NSs and show the residuals of spin period and spin period derivative for different initial values of the wobble angle. In Section 3.2, the pulse-width modulations for different initial values of the wobble angle are displayed.

\subsection{Phase modulation}

Following Jones \& Andersson (2001), we assume for simplicity that the pulsar beam is in the same direction as the magnetic dipole moment $\widehat{m}$. Once the dipole moment sweeps through the plane defined by the line of sight and the spin angular momentum, a pulse can be observed. In Fig. 3, we show the geometry of a freely precessing triaxial NS. We denote the polar angle between $\widehat{e}_{\mathrm{Z}}$ and $\widehat{m}$ as $\Theta$. We denote the azimuthal angle between $\widehat{e}_{X}$ and the projection of $\widehat{m}$ on the $\mathrm{X}-\mathrm{Y}$ plane as $\Phi$. It is related to the Euler angles via (Jones \& Andersson 2001)

$\Phi=\phi-\frac{\pi}{2}+\arctan \left(\frac{\cos \psi \sin \chi}{\sin \theta \cos \chi-\sin \psi \sin \chi \cos \theta}\right)$,

where $\chi$ is the magnetic inclination angle between $\widehat{e}_{3}$ and $\widehat{m}$.

The time derivative of $\Phi$ is the instantaneous spin angular frequency of the NS. Its time-averaged value corresponds to the mean spin angular frequency obtained in the observation. Note that for a precessing triaxial NS, all of the three Euler angles change with time. Especially, the wobble angle varies in a range from its minimum value $\theta_{\min }$ to the maximum value $\theta_{\max }$. We discuss separately the two situations for $\theta_{\min }>\chi$ and $\theta_{\max }<\chi$ to obtain the timing residual of the precessing NS.

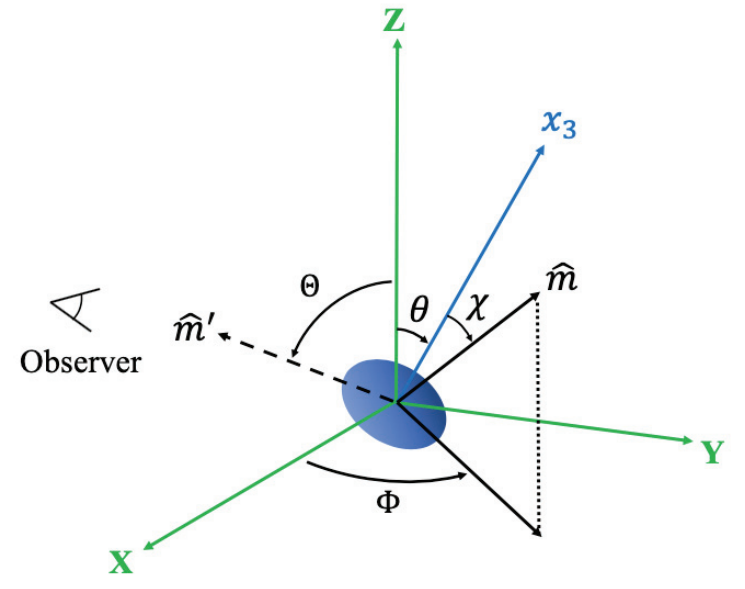

Figure 3. Geometry of a freely precessing triaxial pulsar. The observer is in the $\mathrm{X}-\mathrm{Z}$ plane. The dipole moment is denoted as $\widehat{m}$, and the dashed line $\widehat{m}^{\prime}$ represents the dipole moment when it sweeps through the $\mathrm{X}-\mathrm{Z}$ plane, namely the moment when the observer can see the pulse. We denote the angle between $\widehat{e}_{\mathrm{Z}}$ and $\widehat{m}$ as $\Theta$, and the angle between $\widehat{e}_{3}$ and $\widehat{m}$ (i.e. the magnetic inclination angle) as $\chi$.

(i) When $\theta_{\min }>\chi$, the time-averaged spin frequency of the NS is $\dot{\phi}$. Therefore, the precession-induced phase residual is (Jones \& Andersson 2001)

$\Delta \Phi=\Phi-\left(\phi-\frac{\pi}{2}\right)=\arctan \left(\frac{\cos \psi \sin \chi}{\sin \theta \cos \chi-\sin \psi \sin \chi \cos \theta}\right)$.

(ii) When $\theta_{\max }<\chi$, the time-averaged spin frequency is $\dot{\phi}+\dot{\psi}$, and the precession-induced phase residual is (Jones \& Andersson 2001)

$$
\begin{aligned}
\Delta \Phi & =\Phi-(\phi+\psi) \\
& =\arctan \left[\frac{(\cos \theta-1) \sin \psi \sin \chi-\sin \theta \cos \chi}{\cos \psi \sin \chi+(\cos \theta \sin \psi \sin \chi-\sin \theta \cos \chi) \tan \psi}\right] .
\end{aligned}
$$

The precession-induced residuals of the spin period, $P$, and the spin period derivative, $\dot{P}$, can be calculated using the time derivatives of the precession-induced phase residual,

$\Delta P=-\frac{P_{0}^{2}}{2 \pi} \Delta \dot{\Phi}$,
$\Delta \dot{P}=-\frac{P_{0}^{2}}{2 \pi} \Delta \ddot{\Phi}$,

where $P_{0}$ is the mean spin period of the NS. Substituting the timeevolving Euler angles into equations (50)-(53), one can obtain the modulations of the spin period and the spin period derivative for different choices of parameters.

In Figs 4 and 5, we respectively present examples for cases of a large wobble angle where $\theta_{\min }>\chi$, and a small wobble angle where $\theta_{\max } \ll \chi$. In the calculation, we take the magnetic inclination angle $\chi=\pi / 6$, the mean spin period $P_{0}=0.01 \mathrm{~s}$, and we make use of equation (39) to estimate the oblateness.

(i) The residuals of the spin period and the spin period derivative for the case of a large wobble angle are displayed in the upper panel of Fig. 4. The Fourier transformation of the spin period residual is shown in the lower panel of Fig. 4. The spectrum shows strong peaks 

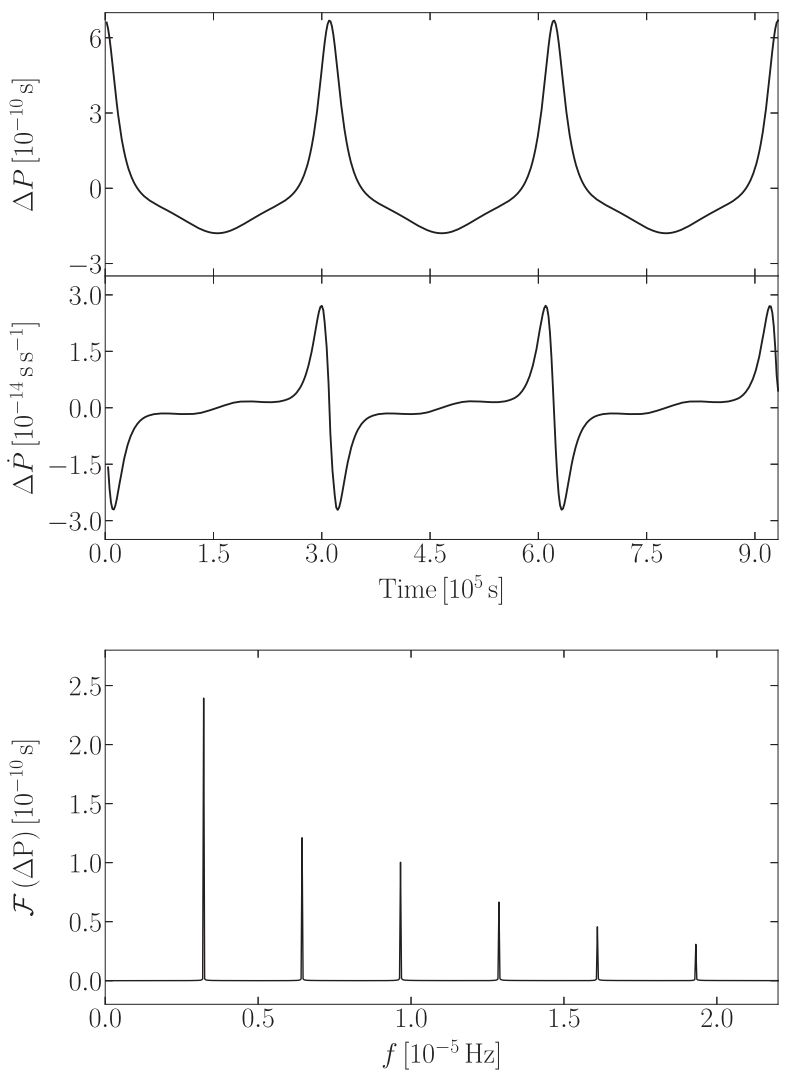

Figure 4. Upper panel: precession-induced residuals of the spin period and the spin period derivative for a large wobble angle where $\theta_{\min }>\chi$. Lower panel: the Fourier amplitude for the spin period residual. In this figure, we have chosen the magnetic inclination angle $\chi=\pi / 6$, the oblateness $\epsilon=$ $4.9 \times 10^{-8}$, the non-axisymmetry parameter $\delta=0.1$, and a wobble angle in the range $\theta \in(0.79,0.84)$. With these parameters, we have $T_{1}=0.010 \mathrm{~s}$, and a free precession period $T=3.1 \times 10^{5} \mathrm{~s}$.

at frequencies $n \Omega_{\mathrm{p}} / 2 \pi$, where $n$ is a positive integer number and $\Omega_{\mathrm{p}}$ $\left(\simeq 2.0 \times 10^{-5} \mathrm{~s}^{-1}\right)$ is the free precession angular frequency defined in equation (20).

(ii) For the small wobble angle limit where $\theta_{\max } \ll \chi$, we display the residuals of the spin period and the spin period derivative in the upper panel of Fig. 5. We also take the Fourier transformation of the spin period residual, whose amplitude is shown in the lower panel of Fig. 5. Notice that a logarithmic scale is used for the Fourier amplitude. Compared to the case of a large wobble angle, the harmonics at $n \Omega_{\mathrm{p}} / 2 \pi(n \geq 2)$ are much weaker than the line at $\Omega_{\mathrm{p}} / 2 \pi$ (now, $\Omega_{\mathrm{p}} \simeq 3.0 \times 10^{-5} \mathrm{~s}^{-1}$ ) in the case of a small wobble angle. In the small wobble angle limit, the precession-induced spin phase residual can be approximated as (Jones \& Andersson 2001; Link \& Epstein 2001)

$\Delta \Phi=-\sin \theta \cot \chi \cos \psi-\frac{1}{4} \sin ^{2} \theta\left(1+2 \cot ^{2} \chi\right) \sin 2 \psi$.

Applying the series expansion of the Euler angles in equations (43)(48) and using equation (51), the spin period residual is given by

$$
\begin{aligned}
\Delta P \approx & \frac{P_{0}^{2}}{2 \pi} \Omega_{\mathrm{p}} \gamma(8 \kappa+1) \cot \chi \cos \left(\Omega_{\mathrm{p}} t\right) \\
& +\frac{P_{0}^{2}}{4 \pi} \Omega_{\mathrm{p}} \gamma^{2}\left(1+2 \cot ^{2} \chi\right) \cos \left(2 \Omega_{\mathrm{p}} t\right) .
\end{aligned}
$$
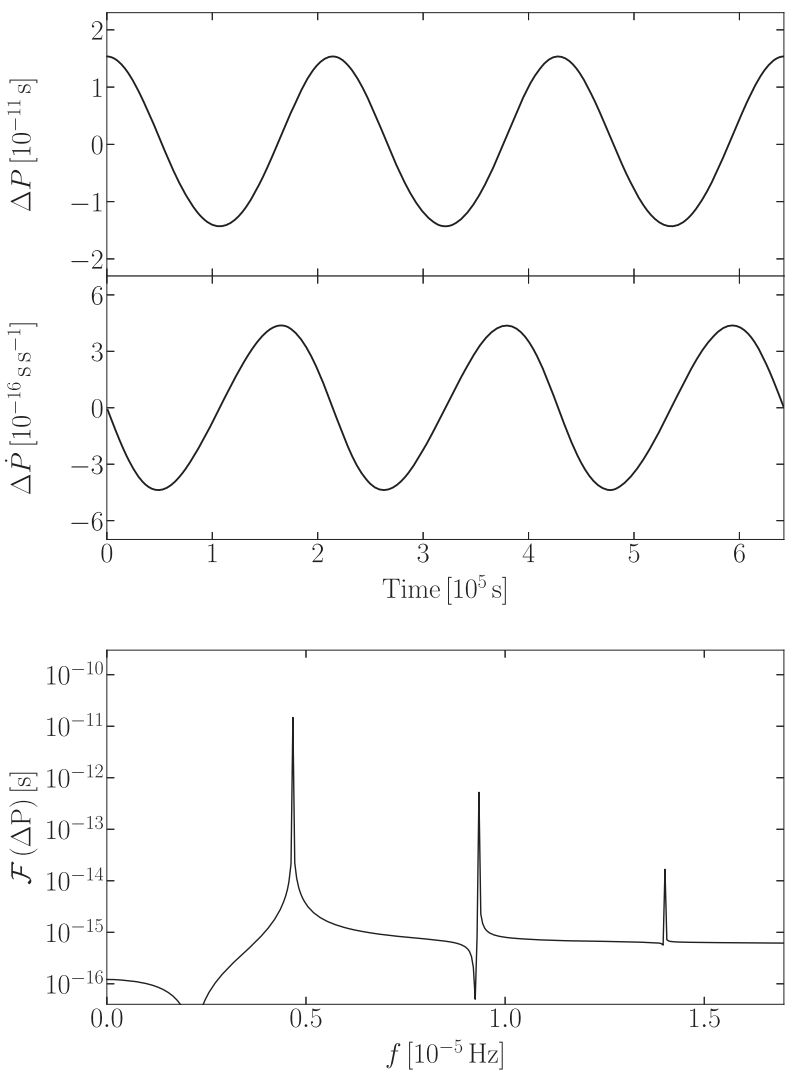

Figure 5. Upper panel: precession-induced residuals of the spin period and the spin period derivative for a small wobble angle where $\theta_{\max } \ll \chi$. Lower panel: the Fourier amplitude for the spin period residual. In this figure, we have chosen the same $\chi, \epsilon$, and $\delta$ as in Fig. 4, but a small wobble angle in the range $\theta \in(0.017,0.018)$. With these parameters, we have $T_{1}=0.010 \mathrm{~s}$, and a free precession period $T=2.1 \times 10^{5} \mathrm{~s}$.

It shows that at the second order of the wobble angle, the modulation of the spin period includes the first and the second harmonics of the free precession angular frequency $\Omega_{\mathrm{p}}$, corresponding to the first two peaks in the lower panel of Fig. 5. The third peak comes from higher order terms that are not included in the approximation. The residual of the spin period derivative $\Delta \dot{P}$ can be obtained by taking the time derivative of $\Delta P$, which gives

$$
\begin{aligned}
\Delta \dot{P} \approx & -\frac{P_{0}^{2}}{2 \pi} \Omega_{\mathrm{p}}^{2} \gamma(8 \kappa+1) \cot \chi \sin \left(\Omega_{\mathrm{p}} t\right) \\
& -\frac{P_{0}^{2}}{2 \pi} \Omega_{\mathrm{p}}^{2} \gamma^{2}\left(1+2 \cot ^{2} \chi\right) \sin \left(2 \Omega_{\mathrm{p}} t\right) .
\end{aligned}
$$

When $\kappa=0$, equations (55) and (56) reduce to the corresponding results for a precessing biaxial NS (Link \& Epstein 2001). Note that we have ignored the intrinsic spin-down of NSs for simplicity in deriving equations (55) and (56).

\subsection{Pulse-width modulation}

In order to analyse the pulse-width modulation, we adopt a simple cone model to describe the radiation of a pulsar. For a more complicated radiation geometry, our method can be extended as well. In the cone model, from the geometry in Fig. 6 we have (Gil et al. 


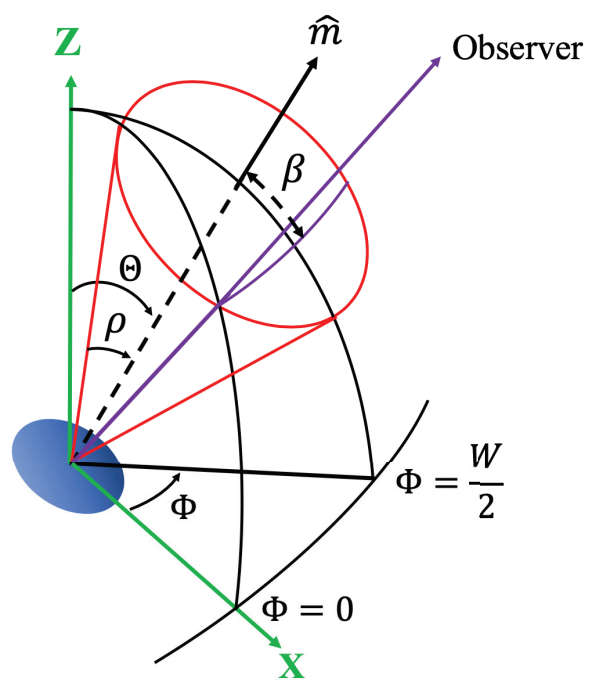

Figure 6. Geometry of the pulsar emission beam in the cone model (Gil et al. 1984; Lorimer \& Kramer 2005). The emission is confined in a cone with an opening angle $\rho$. We denote the impact angle as $\beta$, which corresponds to the closest approach between the line of sight and the magnetic dipole moment. Pulse signals can be observed once the line of sight sweeps through the cone. The purple line denotes the sweep of the line of sight, and different cuts of the line of sight through the cone result in different pulse width $W$.

1984; Lorimer \& Kramer 2005)

$\sin ^{2}\left(\frac{W}{4}\right)=\frac{\sin ^{2}(\rho / 2)-\sin ^{2}(\beta / 2)}{\sin (\Theta+\beta) \sin \Theta}$,

where $\Theta$ is defined in Fig. 3, and $W, \rho$, and $\beta$ are defined in Fig. 6.

Equation (57) is not exact for the pulse width because in our case the angle $\Theta$ changes with time. However, as the spin frequency is much higher than the free precession frequency, the change in $\Theta$ during a spin period is negligible, thus this approximation is good enough for our calculation. In this cone model, the observer can observe the pulse signal once the line-of-sight enters into the emission cone. The variation of pulse width can be determined by the angle $\Theta$ once the inclination angle (denoted as $\iota$ ) between the angular momentum and the line of sight to the NS is determined. The angle $\Theta$ can be expressed through the Euler angles and the magnetic inclination angle via (Jones \& Andersson 2001; Link \& Epstein 2001)

$\cos \Theta=\sin \theta \sin \psi \sin \chi+\cos \theta \cos \chi$.

In Fig. 7, we present examples for the pulse-width modulation with a large wobble angle where $\theta_{\min }>\chi$ and a small wobble angle where $\theta_{\max } \ll \chi$. The choices of the oblateness, the non-axisymmetry, and the magnetic inclination angle are the same as in Figs 4 and 5 for the large and small wobble angles, respectively. The example for the large wobble angle is displayed in the upper panel of Fig. 7. For our (extreme) choice of the parameters, the angle between the angular momentum and the dipole moment changes significantly, $\Theta \in(0.26,1.31)$. As a consequence, the pulse width changes in a wide range with $W \in(0,2.68)$. The line-of-sight leaves out of the emission cone due to the free precession during certain time ranges, and then the pulse width diminishes to zero accordingly. The modulation of pulse width in the case of a small wobble angle is shown in the lower panel of Fig. 7. In this case, the angle $\Theta$ is in the range of $\Theta \in(0.51,0.54)$, and the change of pulse width is much milder with $W \in(2.14,2.21)$.
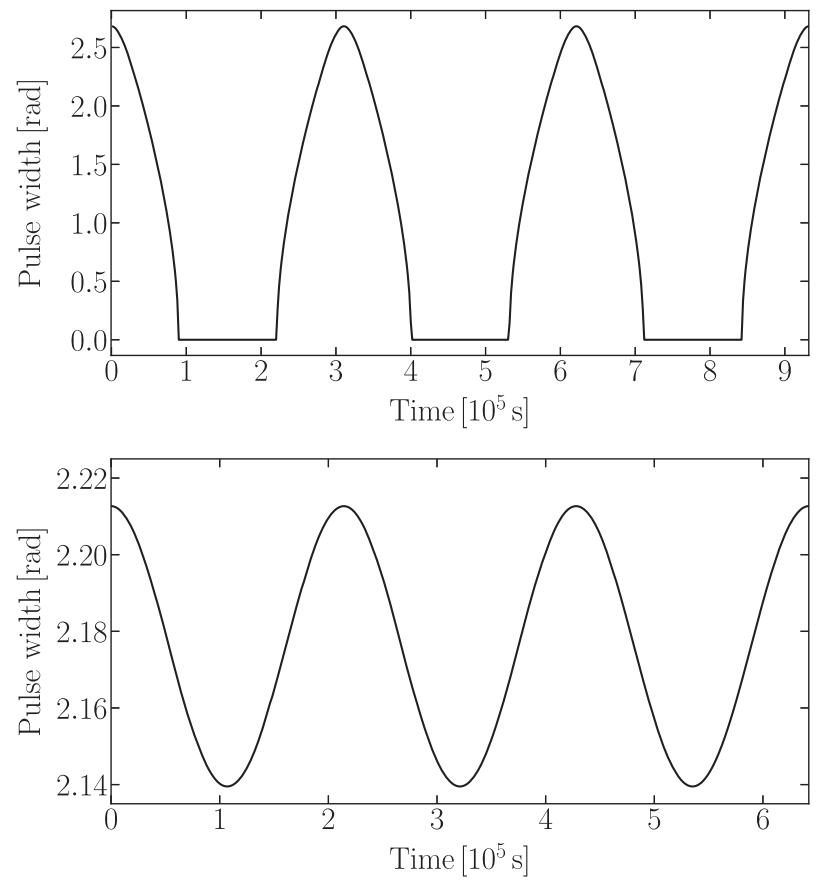

Figure 7. Upper panel: the pulse-width modulation in the case of a large wobble angle; parameters are the same as in Fig. 4. Lower panel: the pulsewidth modulation for a small wobble angle; parameters are the same as in Fig. 5. For both cases, we have chosen the inclination angle $\iota=5 \pi / 6$, and the angular radius of the emission cone $\rho=\pi / 6$.

In the case of a small wobble angle, applying the series expansions of $\theta$ and $\psi$ in equations (43)-(48), the angle $\Theta$ can be approximated as

$\Theta \approx \chi-\sin \theta \sin \psi \approx \chi-\gamma \cos \left(\Omega_{\mathrm{p}} t\right)$,

which reduces to the corresponding result for a precessing biaxial NS (Link \& Epstein 2001).

\section{CONTINUOUS GRAVITATIONAL WAVES}

We discuss generic continuous GWs from a triaxially deformed freely precessing NS in Section 4.1, and the approximation to the waveform with small oblateness, a small wobble angle, and small non-axisymmetry in Section 4.2. The results mainly follow Zimmermann (1980) and Van Den Broeck (2005), but we make further extensions by assuming no hierarchy in the three small parameters.

\subsection{Generic waveform}

We use the quadrupole approximation for the continuous GWs from freely precessing triaxial NSs. In the transverse-traceless (TT) gauge, the metric perturbation is (Misner et al. 1973)

$h_{i j}^{\mathrm{TT}}=\frac{2 G}{r c^{4}} \frac{\mathrm{d}^{2} I_{i j}}{\mathrm{~d} t^{2}}$,

where $G$ is the gravitational constant, $c$ is the speed of light, $I_{i j}$ is the trace-free part of the moment of inertia tensor, and $r$ is the luminosity distance from the source to the observer. 


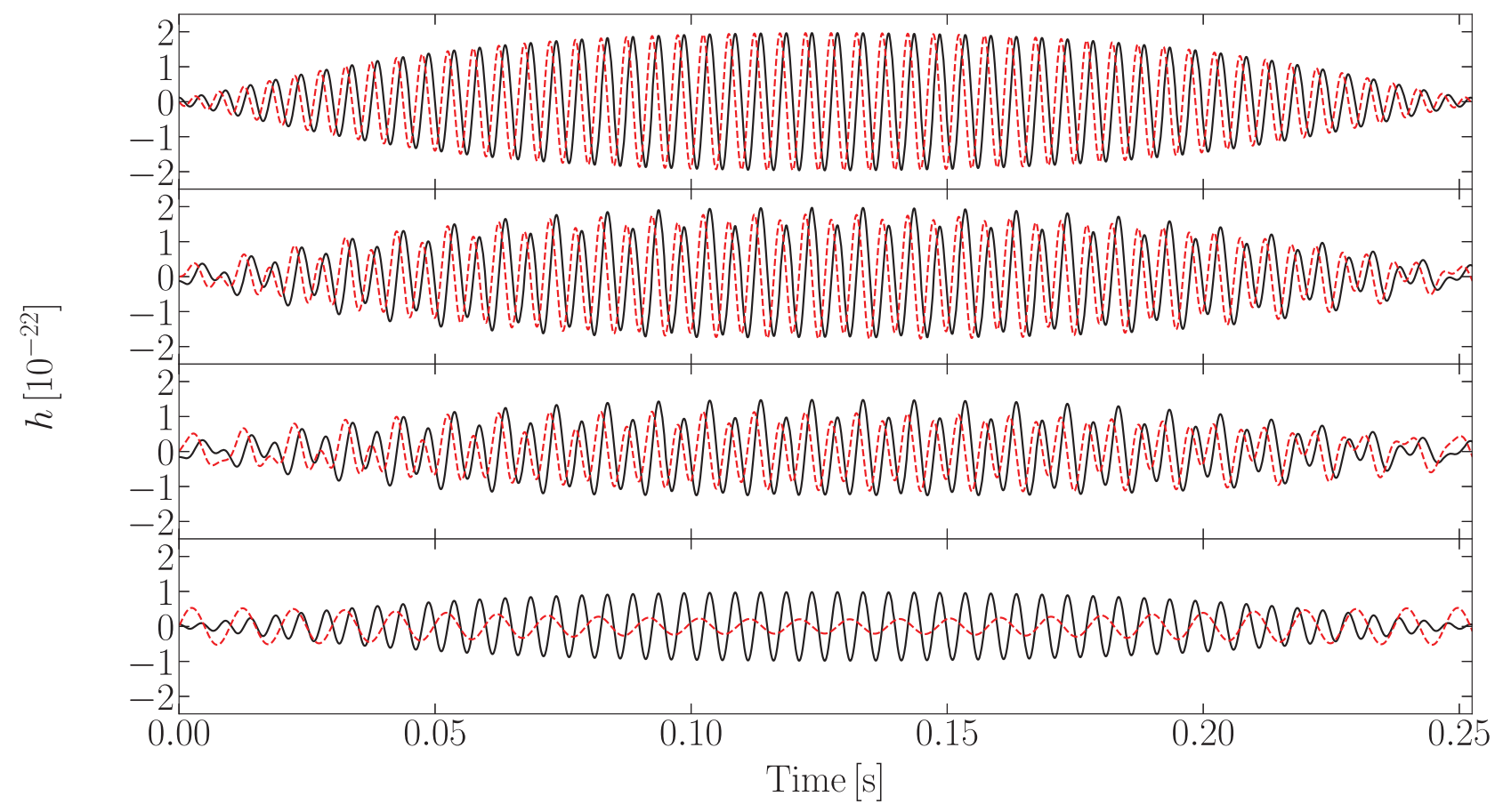

Figure 8. Gravitational waveforms of $h_{+}$(black solid line) and $h_{\times}$(red dotted line) as a function of time at inclination angles (from top to bottom) $\iota=0$, $\pi / 6, \pi / 3$, and $\pi / 2$. We have chosen the oblateness $\epsilon=0.05$, the non-axisymmetry $\delta=0.8$, the wobble angle in the range $\theta \in(0.76,1.21)$, the moment of inertia $I_{3}=10^{45} \mathrm{~g} \mathrm{~cm}^{2}$, and the distance from the observer to the source $r=10 \mathrm{kpc}$. With these exaggerated parameters for illustrative purposes, we have $T_{1}=0.0099 \mathrm{~s}$, and a free precession period $T=0.50 \mathrm{~s}$. This figure contains half of a free precession period.

Alternatively, the quadrupole formula in equation (60) can be expressed as (Zimmermann 1980)

$h_{i j}^{\mathrm{TT}}=-\frac{2 G}{r c^{4}} \mathcal{R}_{i k} \mathcal{R}_{j l} A_{k l}$,

where $\mathcal{R}$ is the rotation matrix (equation 24), and $A_{k l}$ is determined by the body-frame angular velocities and the body-frame moments of inertia. For example, we have

$A_{11}=2\left(\Delta_{2} \omega_{2}^{2}-\Delta_{3} \omega_{3}^{2}\right)$,

$A_{12}=\left(\Delta_{1}-\Delta_{2}+\frac{\Delta_{3}^{2}}{I_{3}}\right) \omega_{1} \omega_{2}$,

where

$\Delta_{1} \equiv I_{2}-I_{3}, \quad \Delta_{2} \equiv I_{3}-I_{1}, \quad \Delta_{3} \equiv I_{1}-I_{2}$.

The other components of $A_{k l}$ can be obtained from equations (62) and (63) by cyclic permutation of the indices.

The waveform in equation (61) is usually decomposed into $h_{+}$and $h_{\times}$,

$h_{i j}^{\mathrm{TT}}=h_{+}\left(\widehat{e}_{+}\right)_{i j}+h_{\times}\left(\widehat{e}_{\times}\right)_{i j}$,

where $h_{+}$and $h_{\times}$represent the radiation of the two independent polarizations. The polarization tensors, $\widehat{e}_{+}$and $\widehat{e}_{\times}$, are

$\widehat{e}_{+}=\widehat{p} \otimes \widehat{p}-\widehat{q} \otimes \widehat{q}$,

$\widehat{e}_{\times}=\widehat{p} \otimes \widehat{q}+\widehat{q} \otimes \widehat{p}$,

where $\widehat{p}$ and $\widehat{q}$ are two unit vectors with $\widehat{p} \times \widehat{q}$ in the propagation direction of the GWs. We assume that the observer lies in the $\mathrm{Y}-\mathrm{Z}$ plane and define the inclination angle $\iota$ as the angle between the direction of the angular momentum $\widehat{e}_{Z}$ and the line of sight to the NS. In the inertial frame, the unit vectors $\widehat{p}$ and $\widehat{q}$ are

$\widehat{p}=-\widehat{e}_{\mathrm{Y}} \cos \iota-\widehat{e}_{\mathrm{Z}} \sin \iota$,

$\widehat{q}=-\widehat{e}_{\mathrm{X}}$

Combining equation (61) and equations (65)-(69), the waveforms of the two polarizations are (Zimmermann 1980; Van Den Broeck 2005)

$h_{+}=-\frac{G}{r c^{4}}\left[\left(\mathcal{R}_{2 k} \cos \iota+\mathcal{R}_{3 k} \sin \iota\right)\left(\mathcal{R}_{2 l} \cos \iota+\mathcal{R}_{3 l} \sin \iota\right)\right.$

$\left.-\mathcal{R}_{1 k} \mathcal{R}_{1 l}\right] A_{k l}$,

$h_{\times}=-\frac{2 G}{r c^{4}}\left(\mathcal{R}_{2 k} \cos \iota+\mathcal{R}_{3 k} \sin \iota\right) \mathcal{R}_{1 l} A_{k l}$.

In Section 2, we have discussed the time evolution of the angular velocities in the body frame and the three Euler angles, so we can obtain $h_{+}$and $h_{\times}$at any given time $t$. In Fig. 8, we plot waveforms of $h_{+}$and $h_{\times}$in the time domain at different inclination angles. Note that the parameters that we have chosen for the plot are exaggerated for NSs for illustrative purposes. Physical parameters consistent with the estimates in Section 2.3 can be easily implemented, but the effects will be too small for visual inspection. We also show the Fourier transformation of the waveforms at the inclination angle of $\iota=$ $\pi / 6$ in Fig. 9. The peaks of the spectra are dominantly at angular frequencies,

$\Omega_{\mathrm{r}}+n \Omega_{\mathrm{p}}, \quad 2 \Omega_{\mathrm{r}}+n \Omega_{\mathrm{p}}$,

where $n=0, \pm 1, \pm 2, \ldots$ is an integer number. 


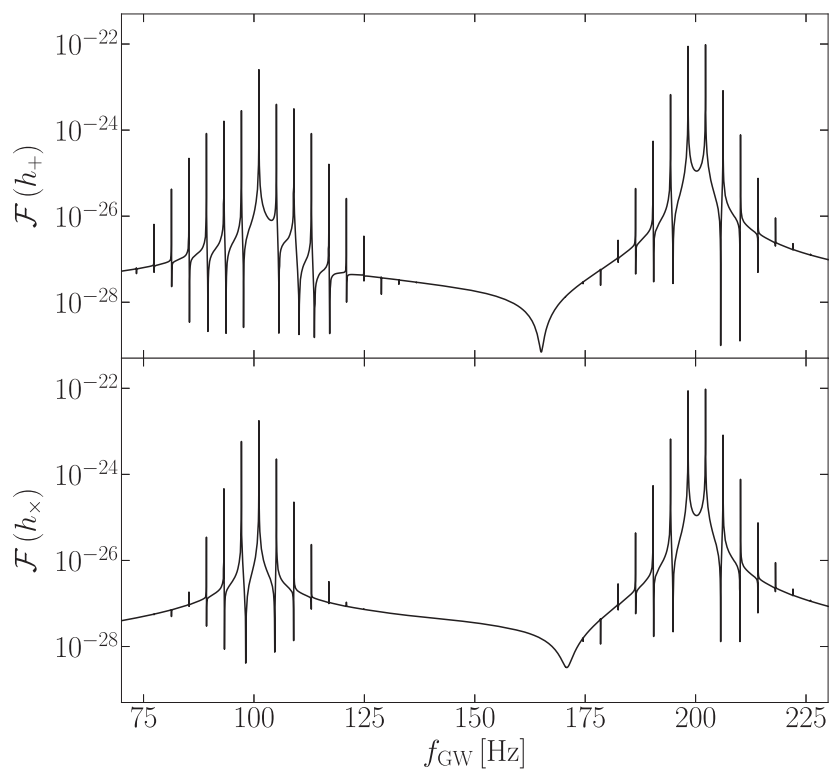

Figure 9. The Fourier amplitudes of $h_{+}$and $h_{\times}$for the waveform in the second panel of Fig. 8 with $\iota=\pi / 6$.

\subsection{Waveform for small oblateness, small wobble angle, and small non-axisymmetry}

Following Zimmermann (1980) and Van Den Broeck (2005), we investigate the waveforms in the limit of small oblateness $\epsilon$, small wobble angle $\theta$, and small non-axisymmetry $\delta$. The difference between our work and the previous work is that, instead of assuming a hierarchy between $\kappa$ and $\gamma$, namely $\kappa \sim \mathcal{O}\left(\gamma^{2}\right)$ in Van Den Broeck (2005), we treat $\gamma$ and $\kappa$ as small quantities independent to each other. As discussed in Section 2.3, it is more plausible to assume no intrinsic hierarchy between $\kappa$ and $\gamma$, in particular when the internal structure of NSs is still rather uncertain.

The procedure to derive the expansion of the waveform is as follows. First, we expand $R_{i j}$ and the angular velocities $\omega_{1}, \omega_{2}$, and $\omega_{3}$ to the second order of $\gamma$ and $\kappa$ using the expansion of Euler angles in equations (43)-(48). Second, we substitute the expansion of $R_{i j}$ and $A_{k l}$ into equations (70) and (71). Third, we retain the GW waveform to the second order of $\gamma$ and $\kappa$ and combine the trigonometric functions using trigonometric identities. Such an extension with independent $\kappa$ and $\gamma$ parameters gives us more features than what was discovered before.

With the above procedure, we obtain six components of $h_{+}$, which are distinguished by different GW frequencies,

$$
\begin{aligned}
h_{+}^{1} & =\frac{-G \epsilon \gamma I_{3} b^{2} \sin 2 \iota}{r c^{4}} \cos \left[\left(\Omega_{\mathrm{r}}+\Omega_{\mathrm{p}}\right) t\right], \\
h_{+}^{2} & =\frac{-32 G \epsilon \kappa I_{3} b^{2}\left(1+\cos ^{2} \iota\right)}{r c^{4}} \cos \left[2 \Omega_{\mathrm{r}} t\right], \\
h_{+}^{3} & =\frac{2 G \epsilon\left(64 \kappa^{2}+\gamma^{2}\right) I_{3} b^{2}\left(1+\cos ^{2} \iota\right)}{r c^{4}} \cos \left[2\left(\Omega_{\mathrm{r}}+\Omega_{\mathrm{p}}\right) t\right], \\
h_{+}^{4} & =\frac{-14 G \epsilon \gamma \kappa I_{3} b^{2} \sin 2 \iota}{r c^{4}} \cos \left[\left(\Omega_{\mathrm{r}}-\Omega_{\mathrm{p}}\right) t\right] \\
h_{+}^{5} & =\frac{2 G \epsilon \gamma \kappa I_{3} b^{2} \sin 2 \iota}{r c^{4}} \cos \left[\left(\Omega_{\mathrm{r}}+3 \Omega_{\mathrm{p}}\right) t\right] \\
h_{+}^{6} & =\frac{-128 G \epsilon \kappa^{2} I_{3} b^{2}\left(1+\cos ^{2} \iota\right)}{r c^{4}} \cos \left[2\left(\Omega_{\mathrm{r}}-\Omega_{\mathrm{p}}\right) t\right] .
\end{aligned}
$$

Similarly, we obtain six components of $h_{\times}$,

$h_{\times}^{1}=\frac{2 G \epsilon \gamma I_{3} b^{2} \sin \iota}{r c^{4}} \sin \left[\left(\Omega_{\mathrm{r}}+\Omega_{\mathrm{p}}\right) t\right]$,

$h_{\times}^{2}=\frac{64 G \epsilon \kappa I_{3} b^{2} \cos \iota}{r c^{4}} \sin \left[2 \Omega_{\mathrm{r}} t\right]$,

$h_{\times}^{3}=\frac{-4 G \epsilon\left(64 \kappa^{2}+\gamma^{2}\right) I_{3} b^{2} \cos \iota}{r c^{4}} \sin \left[2\left(\Omega_{\mathrm{r}}+\Omega_{\mathrm{p}}\right) t\right]$,

$h_{\times}^{4}=\frac{28 G \epsilon \gamma \kappa I_{3} b^{2} \sin \iota}{r c^{4}} \sin \left[\left(\Omega_{\mathrm{r}}-\Omega_{\mathrm{p}}\right) t\right]$,

$h_{\times}^{5}=\frac{-4 G \epsilon \gamma \kappa I_{3} b^{2} \sin \iota}{r c^{4}} \sin \left[\left(\Omega_{\mathrm{r}}+3 \Omega_{\mathrm{p}}\right) t\right]$,

$h_{\times}^{6}=\frac{256 G \epsilon \kappa^{2} I_{3} b^{2} \cos \iota}{r c^{4}} \sin \left[2\left(\Omega_{\mathrm{r}}-\Omega_{\mathrm{p}}\right) t\right]$.

Note that the components with frequencies $\Omega_{\mathrm{r}}+\Omega_{\mathrm{p}}$ and $2 \Omega_{\mathrm{r}}$ are the leading order contributions found in Zimmermann (1980), and that the one with frequency $2\left(\Omega_{\mathrm{r}}+\Omega_{\mathrm{p}}\right)$ is the third spectral line in Van Den Broeck (2005) once $\kappa$ is treated as $O\left(\gamma^{2}\right)$.

Below we briefly discuss the waveform for different choices of $\gamma$ and $\kappa$, and for presentation reasons, we leave their observational aspects to the next section, together with the possible radio/X-ray counterparts.

(i) When $\gamma=0$ and $\kappa \neq 0$, the NS does not precess and GWs are radiated at twice of the rotation frequency. The radiation is caused by the asymmetry between $I_{1}$ and $I_{2}$.

(ii) When $\kappa=0$ and $\gamma \neq 0$, GWs are radiated at $\Omega_{\mathrm{r}}+\Omega_{\mathrm{p}}$ and $2\left(\Omega_{\mathrm{r}}+\Omega_{\mathrm{p}}\right)$. This is the classical result of a precessing biaxial NS (Zimmermann \& Szedenits 1979).

(iii) When $\kappa \neq 0$ and $\gamma \neq 0$, the NS is a precessing triaxial body. At the first order of $\gamma$ and $\kappa$, continuous GWs are emitted at angular frequencies of $\Omega_{\mathrm{r}}+\Omega_{\mathrm{p}}$ and $2 \Omega_{\mathrm{r}}$ (Zimmermann 1980). Previously, Van Den Broeck (2005) treated $\kappa$ as small as $\gamma^{2}$ and got a new line. It is at the second order of $\gamma$, but still at the first order of $\kappa$. The continuous GWs corresponding to this spectral line have an angular frequency of $2\left(\Omega_{\mathrm{r}}+\Omega_{\mathrm{p}}\right)$. Here in our work, we treat $\kappa$ and $\gamma$ independently and expand the waveform to the second order that includes $\gamma^{2}, \kappa^{2}$, and $\gamma \kappa$. We find three new spectral lines at angular frequencies of $\Omega_{\mathrm{r}}-\Omega_{\mathrm{p}}, \Omega_{\mathrm{r}}+3 \Omega_{\mathrm{p}}$, and $2\left(\Omega_{\mathrm{r}}-\Omega_{\mathrm{p}}\right)$. We consider it a natural extension of the results in Van Den Broeck (2005).

\section{DISCUSSIONS}

The first detection of the coalescence of a binary NS system opened the avenue for multimessenger astrophysics (Abbott et al. 2017 b,c,d). In this paper, we discuss another possibility to achieve multimessenger observation with electromagnetic and GW detectors, namely the observation of precessing NSs. Multimessenger astrophysics can be greatly advanced if a precessing NS is observed as a pulsar via radio and/or X-ray telescopes, and in the meantime, its continuous GW radiation is detected by the kilohertz $(\mathrm{kHz})$ laserinterferometric GW detectors, including LIGO (Aasi et al. 2015), Virgo (Acernese et al. 2015), and Kamioka Gravitational Wave Detector (KAGRA; Akutsu et al. 2019). As we will see below, this should surely provide invaluable constraints on the NS structure, complementary to traditional observables, including masses, radii, and tidal deformabilities of NSs.

Radio/X-ray signals and continuous GWs from precessing triaxial NSs will provide valuable information about the wobble angle, the non-axisymmetry, and the oblateness of the source. These measurements are ultimately related to the long-standing question on 
the equation of state for supranuclear matters inside NSs (Lattimer \& Prakash 2001). Below we take the case of a small wobble angle as an example to discuss the extraction of physical properties from such measurements (Van Den Broeck 2005).

For pulsar signals, the amplitude of the spin period residual in equation (55) at the frequencies of $\Omega_{\mathrm{p}}$ and $2 \Omega_{\mathrm{p}}$ can be expressed as

$$
\begin{aligned}
\Delta P_{1}= & 1.6 \times 10^{-10} \cot \chi\left(\frac{P_{0}}{0.01 \mathrm{~s}}\right)^{2}\left(\frac{\Omega_{\mathrm{p}}}{10^{-5} \mathrm{rad} \mathrm{s}^{-1}}\right) \\
& \times(\gamma+8 \kappa \gamma) \mathrm{s}, \\
\Delta P_{2}= & 8.0 \times 10^{-11}\left(1+2 \cot ^{2} \chi\right)\left(\frac{P_{0}}{0.01 \mathrm{~s}}\right)^{2} \\
& \times\left(\frac{\Omega_{\mathrm{p}}}{10^{-5} \mathrm{rad} \mathrm{s}^{-1}}\right) \gamma^{2} \mathrm{~s},
\end{aligned}
$$

where $\Delta P_{1}$ is the amplitude of the spin period residual at the frequency $\Omega_{\mathrm{p}}$, and $\Delta P_{2}$ is the amplitude of the spin period residual at the frequency $2 \Omega_{\mathrm{p}}$.

The elliptic integral of the first kind $K(m)$ approaches $\pi / 2$ in the small-wobble-angle and small-non-axisymmetry limit, which leads to $\Omega_{\mathrm{p}} \rightarrow \epsilon \Omega_{\mathrm{r}}$. Therefore, in such a limiting case, the precession angular frequency $\Omega_{\mathrm{p}}$ can be approximated as (Van Den Broeck 2005)

$\Omega_{\mathrm{p}} \simeq \frac{\pi}{2 K(m)} \epsilon \Omega_{\mathrm{r}}$.

The free precession period $T$ can be directly obtained from the positions of spectral lines in the frequency domain of timing residuals. The wobble angle $\gamma$, the non-axisymmetry $\kappa$, and the magnetic inclination angle $\chi$ cannot be fully determined with two spectral lines. But if the non-axisymmetry is small enough, the second-order contribution to the amplitude of the first line can be ignored. Then the wobble angle $\gamma$ and the magnetic inclination angle $\chi$ can be determined.

The pulse-width modulations will provide important information on the beam shape of pulsars. In this work, we used a simple cone model (Gil et al. 1984) to describe the modulations of pulse width. We find that up to the second order, the pulse-width modulation is the same as that in the biaxial case. From the perspective of observation, if the pulse-width variations from precessing NSs are observed, the beam shape can be inferred via different cuts by the line of sight (Link \& Epstein 2001).

From above discussions, we find that the inclusion of the nonaxisymmetry of NS only slightly changes the timing residuals and the pulse width compared with the biaxial results. The reason is that the parameter $m=16 \kappa \gamma^{2}$ plays an important role in determining the behaviour of free precession. As $m$ approaches zero, the biaxial approximation is robust. Even for a large non-axisymmetry, if the wobble angle $\gamma \ll 1$, the dynamics of the NS still only deviates from the biaxial one slightly. In the case of large wobble angles and large non-axisymmetries, the parameter $m$ can be of order unity. Then the amplitudes of the harmonics are correspondingly large in timing residuals. In this case, if the angle between the beam and the line of sight changes during the free precession, the observer might lose the radiating beam when the line of sight does not cut the radiating region (see the upper panel of Fig. 7). Some pulsars display episodes of interpulsing (i.e. a pulse occurring midway between successive main pulses). The existence of interpulses in a specific system in principle leads to a constraint on the orientation between the magnetic and rotation axes (Akgun et al. 2006), which may give us more information on the pulsar geometries and pulse profiles. More exploration along this line is worthwhile.

For active pulsars, magnetospheric processes may affect the pulse signals from precessing NSs and make the interpretation of free precession complicated. For example, the precession may itself introduce changes on the emission geometry and the activities of the magnetosphere (Link \& Epstein 2001). Besides, the changes of the emission height can contribute to pulse arrival time (Link \& Epstein 2001). Depending on the properties of the observed pulsars, these complications need to be considered. Compared to the radio signals, the X-ray signals are hardly affected by dispersion and scattering during the propagation. The Neutron star Interior Composition Explorer (NICER) mission can give phase-resolved X-ray spectroscopy for pulsars (Bilous et al. 2019; Riley et al. 2019). High-precision Xray timing for millisecond pulsars has also been conducted (Deneva et al. 2019). In the near future, NICER X-ray timing of pulsars may help to track the precession of NSs better.

As a new observation window, GWs from precessing NSs can give complementary physical information on these triaxial NSs. Following Van Den Broeck (2005), we present the procedures to extract physical parameters from continuous GWs. We take the ' $x$ ' mode as an example, and the discussion for the ' + ' mode is similar. For the ' $X$ '-polarized GW, the amplitudes of the first-order lines at $\Omega_{\mathrm{r}}+\Omega_{\mathrm{p}}$ and $2 \Omega_{\mathrm{r}}$ are

$$
A_{\times}^{1}=1.0 \times 10^{-28} \gamma \sin \iota\left(\frac{\epsilon}{4.9 \times 10^{-8}}\right)\left(\frac{f_{\mathrm{r}}}{100 \mathrm{~Hz}}\right)^{2}\left(\frac{10 \mathrm{kpc}}{r}\right),
$$

$A_{\times}^{2}=3.3 \times 10^{-27} \kappa \cos \iota\left(\frac{\epsilon}{4.9 \times 10^{-8}}\right)\left(\frac{f_{\mathrm{r}}}{100 \mathrm{~Hz}}\right)^{2}\left(\frac{10 \mathrm{kpc}}{r}\right)$,

where we have assumed that the moment of inertia of the NS is $10^{45} \mathrm{~g} \mathrm{~cm}^{2}$ and applied equation (39) to estimate the oblateness at specific rotation frequency for a NS with $M=1.4 \mathrm{M}_{\odot}$ and $R=$ $10 \mathrm{~km}$.

If the first-order lines are observed, the rotation frequency and the free precession frequency can be determined. The inclination angle $\iota$ can be obtained by comparing the amplitudes of different polarizations for the two first-order lines. Note that the determination of the inclination angle $\iota$ is model dependent with the radio signals (Jones 2007). The derived ८ from continuous GWs is less model dependent and can help to probe the pulsar geometry (Jones 2007). In this work, the inclination angle $\iota$ is needed to determine the pulsewidth modulations.

The oblateness, non-axisymmetry, and wobble angle are degenerate in the first-order waveform. For the ' $x$ '-polarized GW, the amplitudes of the second-order lines at $2\left(\Omega_{\mathrm{r}}+\Omega_{\mathrm{p}}\right), \Omega_{\mathrm{r}}-\Omega_{\mathrm{p}}, \Omega_{\mathrm{r}}+$ $3 \Omega_{\mathrm{p}}$, and $2\left(\Omega_{\mathrm{r}}-\Omega_{\mathrm{p}}\right)$ are, respectively,

$$
\begin{aligned}
A_{\times}^{3}= & -2.1 \times 10^{-28}\left(64 \kappa^{2}+\gamma^{2}\right) \\
& \times \cos \iota\left(\frac{\epsilon}{4.9 \times 10^{-8}}\right)\left(\frac{f_{\mathrm{r}}}{100 \mathrm{~Hz}}\right)^{2}\left(\frac{10 \mathrm{kpc}}{r}\right), \\
A_{\times}^{4}= & 1.5 \times 10^{-27} \gamma \kappa \sin \iota\left(\frac{\epsilon}{4.9 \times 10^{-8}}\right)\left(\frac{f_{\mathrm{r}}}{100 \mathrm{~Hz}}\right)^{2} \\
& \times\left(\frac{10 \mathrm{kpc}}{r}\right),
\end{aligned}
$$




$$
\begin{aligned}
A_{\times}^{5}= & -2.1 \times 10^{-28} \gamma \kappa \sin \iota\left(\frac{\epsilon}{4.9 \times 10^{-8}}\right)\left(\frac{f_{\mathrm{r}}}{100 \mathrm{~Hz}}\right)^{2} \\
& \times\left(\frac{10 \mathrm{kpc}}{r}\right), \\
A_{\times}^{6}= & 1.3 \times 10^{-26} \kappa^{2} \cos \iota\left(\frac{\epsilon}{4.9 \times 10^{-8}}\right)\left(\frac{f_{\mathrm{r}}}{100 \mathrm{~Hz}}\right)^{2} \\
& \times\left(\frac{10 \mathrm{kpc}}{r}\right) .
\end{aligned}
$$

Theoretically, by comparing the amplitudes of the two first-order lines and one of the second-order lines, the wobble angle $\gamma$ and the non-axisymmetry $\kappa$ can be determined. Then, one can obtain the parameter $m=16 \kappa \gamma^{2}$ so that the oblateness can be determined using equation (87).

From the observational perspective, however, these amplitudes at the second order are very small, and unlikely to be detectable with the Advanced LIGO/Virgo detectors. Besides, if the coherent time of the observation is shorter than the free precession period, the free precession angular frequency $\Omega_{\mathrm{p}}$ cannot be resolved in frequency domain. However, in the optimistic situation when they are observed with the next-generation GW detectors (e.g. the Einstein Telescope and Cosmic Explorer; Punturo et al. 2010; Hild et al. 2011; Sathyaprakash et al. 2012; Abbott et al. 2017a), they can be used to infer the oblateness, non-axisymmetry, and wobble angle of the star. The distance to the NS and the moment of inertia always enter the waveform through the combination $I_{3} / r$. Therefore, we cannot obtain them independently. By inserting an educated guess of $I_{3}$, the distance to the NS can be roughly determined (Van Den Broeck 2005). Or conversely, if the distance can be determined via parallax or dispersion measure in pulsar timing data, one can get a measurement of $I_{3}$, thus putting new constraints on the equation of state. Detailed analysis along this line is beyond the scope of this paper, and we leave it to future study.

\section{SUMMARY}

To summarize, in this paper we describe both the analytical and numerical methods to calculate the dynamical evolution of precessing triaxial rigid bodies. We discuss the timing residuals and the pulsewidth modulations for precessing triaxial NSs. We also present concrete examples of the timing residuals and the pulse-width modulations for large and small wobble angles. For the GWs from triaxial precessing NSs, after reviewing the general solution of the quadrupole waveform (Zimmermann 1980) and showing examples of the waveform in both time and frequency domains, we extend the work by Van Den Broeck (2005) at the second order by relaxing the assumption on the small parameters $\gamma$ and $\kappa$. We obtain three new lines in the continuous GWs spectra, which might be useful for future continuous $\mathrm{GW}$ analysis using the third-generation groundbased detectors (Abbott et al. 2017a), depending on the distance of the sources. If the prospects of the multimessenger astrophysics discussed in this work become reality, numerous information on the shape of NSs and the equation of state of supranuclear matters will be obtained, enabling a new frontier for fundamental physics.

\section{ACKNOWLEDGEMENTS}

We thank the anonymous referee for helpful comments and suggestions. This work was supported by the National Natural Science Foundation of China (11975027, 11991053, 11721303, and
11673002), the Young Elite Scientists Sponsorship Program by the China Association for Science and Technology (2018QNRC001), the Max Planck Partner Group Program funded by the Max Planck Society, and the High-performance Computing Platform of Peking University. It was partially supported by the Strategic Priority Research Program of the Chinese Academy of Sciences through the Grant No. XDB23010200. L. Sun is a member of the LIGO Laboratory. LIGO was constructed by the California Institute of Technology and Massachusetts Institute of Technology with funding from the United States National Science Foundation, and operates under cooperative agreement PHY-1764464. Advanced LIGO was built under award PHY-0823459.

\section{DATA AVAILABILITY}

The data underlying this paper will be shared on reasonable request to the corresponding author.

\section{REFERENCES}

Aasi J. et al., 2015, Classical Quantum Gravity, 32, 074001

Abbott B. P. et al., 2016, Phys. Rev. Lett., 116, 061102

Abbott B. P. et al., 2017a, Classical Quantum Gravity, 34, 044001

Abbott B. P. et al., 2017b, Phys. Rev. Lett., 119, 161101

Abbott B. P. et al., 2017c, ApJ, 848, L12

Abbott B. P. et al., 2017d, ApJ, 848, L13

Abbott B. P. et al., 2019a, Phys. Rev. X, 9, 031040

Abbott B. P. et al., 2019b, ApJ, 879, 10

Acernese F. et al., 2015, Classical Quantum Gravity, 32, 024001

Akgun T., Link B., Wasserman I., 2006, MNRAS, 365, 653

Akutsu T. et al., 2019, Nat. Astron., 3, 35

Alpar M., Pines D., 1985, Nature, 314, 334

Amiri M. et al., 2020, Nature, 582, 351

Arribas M., Elipe A., Palacios M., 2006, Celest. Mech. Dyn. Astron., 96, 239

Baiko D., Chugunov A., 2018, MNRAS, 480, 5511

Baym G., Pines D., 1971, Ann. Phys., 66, 816

Baym G., Hatsuda T., Kojo T., Powell P. D., Song Y., Takatsuka T., 2018, Rep. Progress Phys., 81, 056902

Betsch P., Siebert R., 2009, Int. J. Numer. Methods Eng., 79, 444

Bilous A. V. et al., 2019, ApJ, 887, L23

Bisnovatyi-Kogan G. S., Mersov G. A., Sheffer E. K., 1990, SvA, 34, 44

Cordes J. M., 1993, in Phillips J. A., Thorsett S. E., Kulkarni S. R., eds, ASP Conf. Ser. Vol. 36, Planets Around Pulsars. Astron. Soc. Pac., San Francisco, p. 43

Coutsias E. A., Romero L., 2004, The Quaternions with an Application to Rigid Body Dynamics. Sandia National Laboratories, Albuquerque, NM

Cutler C., Jones D. I., 2001, Phys. Rev. D, 63, 024002

Cutler C., Ushomirsky G., Link B., 2003, ApJ, 588, 975

Dai Z., Lu T., 1998, A\&A, 333, L87

Deneva J. et al., 2019, ApJ, 874, 160

Desvignes G. et al., 2019, Science, 365, 1013

Gil J., Gronkowski P., Rudnicki W., 1984, A\&A, 132, 312

Goldreich P., 1970, ApJ, 160, L11

Hild S. et al., 2011, Classical Quantum Gravity, 28, 094013

Horowitz C., Kadau K., 2009, Phys. Rev. Lett., 102, 191102

Janssen G. et al., 2015, PoS, AASKA14, 037

Jones D. I., 2007, Ap\&SS, 308, 125

Jones D. I., Andersson N., 2001, MNRAS, 324, 811

Jones D. I., Andersson N., 2002, MNRAS, 331, 203

Landau L. D., Lifshitz E. M., 1960, Mechanics. Pergamon Press, Oxford

Lasky P. D., Melatos A., 2013, Phys. Rev. D, 88, 103005

Lattimer J., Prakash M., 2001, ApJ, 550, 426

Levin Y., Beloborodov A. M., Bransgrove A., 2020, ApJ, 895, L30

Link B., 2003, in Bailes M., Nice D. J., Thorsett S. E., eds, ASP Conf. Ser. Vol. 302, Radio Pulsars. Astron. Soc. Pac., San Francisco, p. 241

Link B., Epstein R. I., 2001, ApJ, 556, 392 
Link B., Franco L. M., Epstein R. I., 1998, ApJ, 508, 838

Lorimer D. R., Kramer M., 2005, Handbook of Pulsar Astronomy. Cambridge Univ. Press, Cambridge

Mannarelli M., Rajagopal K., Sharma R., 2007, Phys. Rev. D, 76, 074026

Misner C. W., Thorne K. S., Wheeler J. A., 1973, Gravitation. Freeman \& Co., San Francisco

Olver F. W., Lozier D. W., Boisvert R. F., Clark C. W., 2010, NIST Handbook of Mathematical Functions. Cambridge Univ. Press, Cambridge

Owen B. J., 2005, Phys. Rev. Lett., 95, 211101

Perera B. B. P. et al., 2019, MNRAS, 490, 4666

Pina E. G., 2015, J. Geometry Symmetry Phys., 39, 55

Punturo M. et al., 2010, Classical Quantum Gravity, 27, 194002

Riley T. E. et al., 2019, ApJ, 887, L21

Ruderman M., Pines D., Tamagaki R., Tsuruta S., 1992, Structure and Evolution of Neutron Stars. Basic Books, New York

Sathyaprakash B. et al., 2012, Classical Quantum Gravity, 29, 124013

Shabanova T. V., Lyne A. G., Urama J. O., 2001, ApJ, 552, 321

Shakura N., Postnov K., Prokhorov M., 1998, A\&A, 331, L37

Stairs I., Lyne A., Shemar S., 2000, Nature, 406, 484
Suvorov A. G., Kokkotas K. D., 2020, ApJ, 892, L34

Taylor J. H., Fowler L. A., McCulloch P. M., 1979, Nature, 277, 437

Thorne K. S., 1980, Rev. Modern Phys., 52, 299

Van Den Broeck C., 2005, Classical Quantum Gravity, 22, 1825

Wasserman I., 2003, MNRAS, 341, 1020

Whittaker E. T., 1988, A Treatise on the Analytical Dynamics of Particles and Rigid Bodies. Cambridge Univ. Press, Cambridge

Witten E., 1984, Phys. Rev. D, 30, 272

Xu R.-X., 2003, ApJ, 596, L59

Xu R., Zhao J., Shao L., 2020, Phys. Lett. B, 803, 135283

Zanazzi J., Lai D., 2020, ApJ, 892, L15

Zhang B., Fan Y. Z., Dyks J., Kobayashi S., Meszaros P., Burrows D. N., Nousek J. A., Gehrels N., 2006, ApJ, 642, 354

Zimmermann M., 1980, Phys. Rev. D, 21, 891

Zimmermann M., Szedenits E., 1979, Phys. Rev. D, 20, 351

This paper has been typeset from a $\mathrm{T}_{\mathrm{E}} \mathrm{X} / \mathrm{L} \mathrm{T} \mathrm{EX}$ file prepared by the author. 